\title{
The effects of stimulus similarity on different types of behavioral contrast
}

\author{
BEN A. WILLIAMS \\ University of California, San Diego, La Jolla, California
}

\begin{abstract}
Pigeons were trained on multiple schedules with component stimuli of different degrees of similarity. In Experiment 1, a two-component schedule was used in which the two stimuli were either two line orientations or a line orientation versus a diffuse color. Reinforcement rate was varied in one component to determine the effects of stimulus similarity on different aspects of behavioral contrast. Contrast in terms of average response rates (molar contrast) was larger with less similar stimuli. Local contrast effects at the beginning of the component were larger for more similar stimuli, but these effects were more variable and did not attain statistical significance. Independent of the level of molar contrast, the local pattern of schedule interaction differed for the two levels of similarity: with more similar stimuli, the maximum degree of interaction occurred at the beginning of the components and then decreased; with less similar stimuli, the degree of interaction increased throughout the components and was at its maximum near their end. In Experiment 2, the same three stimuli were used while reinforcement rate in the middle component of a three-component sequence was varied; this isolated the effects of the preceding schedule from those of the following schedule. Contrast effects were generally greater in the target component preceding the variable schedule, and these were enhanced by less similar stimuli. Contrast in the target component following the variable schedule was manifested primarily in terms of the behavior at the beginning of the component, and these effects were inconsistently related to stimulus similarity. The functional separation of the effects of stimulus similarity on the different locations of contrast suggest that "anticipatory contrast" and "local contrast" depend upon different mechanisms, thus excluding any account of contrast solely in terms of relative rate of reinforcement.
\end{abstract}

An important distinction in the study of contrast effects in free-operant schedules is that between local versus molar contrast. Molar contrast refers to the average change in response rate as a function of the change in reinforcement rate in the alternative component(s); local contrast refers to the larger than average rate changes in the early portions of the target component (immediately following the transition from the alternative schedule). ${ }^{1}$ Despite considerable previous research, the relationship between local and molar contrast remains uncertain. Some accounts of contrast have claimed that local contrast is an essential part of molar contrast, because the controlling variable is the change in signal value that occurs at the transition between components, which produces elicited responses superimposed on the operant baseline (the "additivity theory" of Rachlin, 1973, and Schwartz \& Gamzu, 1977). In support of such accounts are several studies showing strong parallels between local and molar contrast (Green \& Rachlin, 1975; McLean \& White, 1981; Schwartz, 1978; Spealman, 1976). Other studies, however, have found that local and molar contrast are independent, since local contrast is an important component of molar con-

This research was supported by NSF Grant BNS 84-08878 and NIMH Grant 1 RO1 MH42797 to the University of California, San Diego. Thanks are extended to Paul Royalty for running the subjects and for his comments on an earlier draft of the paper. Reprint requests should be addressed to the author, Department of Psychology, C-009, University of California, San Diego, La Jolla, CA 92093. trast early in training and then disappears while substantial molar contrast still remains (Boneau \& Axelrod, 1962; Mackintosh, Little, \& Lord, 1972; Malone, 1976; Nevin \& Shettleworth, 1966). Such results have encouraged the view that local contrast is the result of Pavlovian processes involved in the formation of a discrimination, which then dissipate as the formation of the discrimination is complete. Such a notion implies that local contrast is not due to the signal value of the onset of the more positive stimulus, but rather to a "release" from the effects of the more negative stimulus that just preceded it. Accordingly, local contrast should be a component of molar contrast during early and intermediate stages of training, on the assumption that the "dynamic" properties of the Sdiminish as the discrimination is learned (cf. Terrace, 1966). Similarly, local contrast should be facilitated by whatever experimental conditions make the discrimination more difficult (see Malone, 1976, for a more complete discussion of the attributes of such Pavlovian processes). Molar contrast, in comparison, is dependent primarily on the relative rates of reinforcement in the components of the multiple schedule, and thus will persist independently of those Pavlovian processes (see Williams, 1983, for a review).

The most frequent method used to study the relationship between local and overall contrast has been an examination of the pattern of local response rates when different rates of reinforcement occur during the two com- 
ponents of the schedule. The pattern expected on the basis of local contrast is a decreasing local rate in the component with the greater frequency of reinforcement and an increasing rate in the component correlated with the lower reinforcement frequency. As noted above, such patterns have frequently been reported early in training but have also been found to disappear with continued exposure to the schedule (e.g., Nevin \& Shettleworth, 1966). However, several recent studies have reported such localrate gradients with steady-state procedures after extended training (McLean \& White, 1981; White, Pipe, \& McLean, 1984; White, Pipe, \& Redman, 1985). Other studies have reported just the opposite type of local-rate gradient, as response rates increased throughout the higher valued component and decreased throughout the lower valued component (Buck, Rothstein, \& Williams, 1975; Hamilton \& Silberberg, 1978; Williams, 1974, 1976a, 1976b). In still other studies, the latter gradients have occasionally occurred in combination with evidence for local contrast, such that a brief elevation at the beginning of the higher valued component was followed by a decrease in rate and then a subsequent increase, thus producing a U-shaped gradient (Williams, 1982; also see Figure 6 of Williams, 1983).

A variable that may account at least partly for the different patterns of local response rates is the similarity between the discriminative stimuli correlated with the different components of the multiple schedule. To the extent that local contrast is the result of the discrimination process involved in diffentiating the two stimuli and is less likely when discrimination is complete, more local contrast should occur with more difficult discriminations. Thus, local contrast might be expected early in training and then disappear with relatively easy discriminations, but persist when the two discriminative stimuli share many features in common. One should expect, therefore, that the degree of local contrast should be greater with high degrees of stimulus similarity. But molar contrast is primarily a function of the relative rate of reinforcement in the different components, and should presumably be greater with less stimulus similarity, because those are the conditions under which the differences between the reinforcement rates in the different components should be most apparent. It should be noted, however, that the degree of molar contrast is partly a function of the amount of local contrast, so the effects of stimulus similarity on molar contrast could vary substantially as a function of the percentage of the total amount of contrast that is due to local contrast effects.

Previous studies of stimulus similarity have yielded conflicting results. Some have reported both larger molar and local contrast effects with greater similarity (Blough, 1980, 1983; Hearst, 1969), whereas others (Mackintosh et al., 1972; Malone, 1976; White, Pipe, \& McLean, 1983; White et al., 1984) have reported just the opposite effect (greater contrast with less similar stimuli). Such disparate results suggest that the function relating stimulus similarity to contrast is nonmonotonic, which typically is due to two separate underlying functional relationships' being combined. Thus, highly similar stimuli might produce relatively little contrast because of stimulus generalization between components, the limiting case being when the two stimuli are identical. Contrast should then increase with greater separation between the discriminative stimuli, both because local contrast begins to occur as some degree of discrimination occurs and because of the increased discrimination of the relative reinforcement rates. Then, however, increasing dissimilarity should eliminate any confusion between the stimuli, thus reducing the contribution of local contrast but enhancing further the size of the contrast effect due solely to relative reinforcement rate. Assuming that local contrast is relatively large in comparison with the simple effects of relative rate of reinforcement, the result would be a nonmonotonic function. In support of this possibility is the fact that some studies that have reported greater local contrast with greater stimulus dissimilarity (Malone, 1976; Mackintosh et al., 1972) have also reported the complete absence of local contrast when the positive stimulus was alternated with periods of time-out, coupled with a generally lower degree of molar contrast than when an explicit $\mathbf{S}-$ was used. To the extent that time-out can be regarded as a cue for extinction $(\mathrm{S}-)$ that is maximally different from a cue for positive reinforcement $(\mathbf{S}+$ ), such effects suggest that the different effects of stimulus similarity previously obtained may, indeed, be due to the varying contribution of local contrast to molar contrast effects.

Previous studies investigating the effects of stimulus similarity on contrast (e.g., White et al., 1983) have varied similarity within a single dimension, most frequently line orientation. Thus, the maximal degree of dissimilarity has been a comparison between two lines differing in orientation by $90^{\circ}$ or between two colors (e.g., red vs. green). To the extent that the function relating local contrast to stimulus similarity is indeed nonmonotonic, this degree of physical separation may not be sufficient to sample both sides around the inflection point of the function. The present study was thus an attempt to study the effects of still greater stimulus differences by using an interdimensional discrimination (vertical lines vs. a diffuse yellow light). This interdimensional discrimination, which should minimize stimulus generalization between the components, was compared with an intradimensional discrimination similar to that used in previous studies (vertical lines vs. horizontal lines). To the extent that local contrast depends on incomplete discrimination between the two discriminative stimuli, the expectation was that greater local contrast should occur with the more similar stimuli. Given such an effect, it then should be possible to assess the role of local contrast as a contributor to molar contrast, and to determine whether its role changes as a function of the degree of stimulus similarity.

\section{EXPERIMENT 1}

\author{
Method \\ Subjects. Four White Carneau pigeons were maintained at $80 \%$ \\ of their free-feeding body weights by additional feeding, when neces-
}


sary, after the experimental sessions. All subjects had had prior training in an undergraduate laboratory, in which they had been trained to peck a diffuse white key under various simple schedules of reinforcement.

Apparatus. A standard conditioning chamber was constructed from a plastic picnic chest. The bird's chamber was approximately $30.5 \mathrm{~cm}$ in all dimensions. On the front panel, which was painted white, were mounted two 1.9 -cm-diam. pigeon keys, which required a minimum force of $0.10 \mathrm{~N}$ for operation. The only feedback for a peck to the key was a click provided by the microswitch that registered key movement and provided the signal to an electromechanical pulseformer. Only the right key was used in the present study. The stimuli were projected onto the rear of the response key by an IEE 28-V 12-stimulus projector. The stimuli were four vertical white lines on a dark background, four horizontal white lines on a dark background, or a diffuse yellow light that transilluminated the entire response key. The line stimuli were $1.7 \mathrm{~cm}$ in length and extended across the entire response key in width. Each individual line was approximately $0.28 \mathrm{~cm}$ in width, with adjacent lines separated by $0.28 \mathrm{~cm}$. As measured by a spot photometer, the brightnesses were $1.2 \mathrm{~cd} / \mathrm{m}^{2}$ for the vertical lines, $0.8 \mathrm{~cd} / \mathrm{m}^{2}$ for the horizontal lines, and $2.6 \mathrm{~cd} / \mathrm{m}^{2}$ for the yellow stimulus. Ten centimeters below the response keys was a $5 \times 5 \mathrm{~cm}$ aperture through which the birds were fed with mixed grain when the food magazine was activated and illuminated by white light. A shielded white houselight, which was illuminated at all times during the session, was located on the rear wall of the chamber opposite the response key.

Procedure. Because all subjects had previously learned to keypeck, they were begun immediately on a multiple schedule with the stimuli shown in Table 1. Throughout training, the two components of the multiple schedule alternated every $30 \mathrm{sec}$ and all subjects received the same schedules. For the first three sessions of training, the schedules during both components were variable interval (VI) $20 \mathrm{sec}$. The schedule during the first component (always the vertical lines) was changed to VI $90 \mathrm{sec}$, and that for the second component was changed to VI $30 \mathrm{sec}$. Two subjects (S-24, S-25) were initially trained with the horizontal lines as the discriminative stimulus for the second component; 2 other subjects (S-26, S-28) were trained initially with yellow as the second discriminative stimulus. After behavior had stabilized during the first condition, the schedule during the second component was changed to VI $270 \mathrm{sec}$. Then, after behavior had again stabilized, the stimulus assignments for the two pairs of birds were reversed while leaving the schedules unchanged. Finally, the stimulus conditions remained unchanged and the schedule during the second component was returned to VI $30 \mathrm{sec}$. A summary of the different conditions for each subject is provided in Table 1 .

Table 1

Outline of the Experimental Conditions Presented to Each Subject in Experiment 1

\begin{tabular}{cclc}
\hline Condition & Subjects & $\begin{array}{c}\text { Component 2 } \\
\text { Stimulus }\end{array}$ & $\begin{array}{c}\text { Component } 2 \\
\text { Schedule }\end{array}$ \\
\hline $1(40)$ & S-24,S-25 & Horizontal lines & VI $30 \mathrm{sec}$ \\
& S-26,S-28 & Yellow & VI $30 \mathrm{sec}$ \\
$2(56)$ & S-24,S-25 & Horizontal lines & VI $270 \mathrm{sec}$ \\
& S-26,S-28 & Yellow & VI 270 sec \\
$3(31)$ & S-24,S-25 & Yellow & VI $270 \mathrm{sec}$ \\
& S-26,S-28 & Horizontal lines & VI 270 sec \\
$4(32)$ & S-24,S-25 & Yellow & VI $30 \mathrm{sec}$ \\
& S-26,S-28 & Horizontal lines & VI $30 \mathrm{sec}$ \\
\hline
\end{tabular}

Note-Shown in parentheses is the number of sessions per condition presented to all subjects. The schedule during Component 1 was always VI $90 \mathrm{sec}$, and the stimulus correlated with Component 1 was always the vertical lines.
All schedules were constructed from the exponential distributions of Fleshler and Hoffman (1962), using 18 different intervals. Reinforcement consisted of 3-sec access to mixed grain, during which time the keylight was darkened. Training within a session continued until 60 presentations of each component had been presented. Training within a condition continued for a minimum of 30 sessions and until all birds had met a stability criterion; the relative response rates for blocks of 3 sessions were calculated, and training was continued until there were no ascending or descending trends for three such blocks and until the difference between the highest and lowest relative rate was less than 0.05 . The large number of sessions presented in Condition 2 was due to an intermittent equipment failure (a loose connection for the light that illuminated the food hopper), which occurred between Sessions 25 and $\mathbf{4 0}$ and resulted in several of the subjects' having to meet the stability criterion a second time.

\section{Results}

Although the different types of discriminative stimuli clearly involved different degrees of similarity for human observers, it is important to demonstrate that the different stimuli did indeed vary in degree of similarity for the pigeons. By convention, similarity is defined by the amount of confusion between the two reinforcement schedules, or conversely by the degree of discrimination. The best assessment of the degree of discrimination between components is a comparison of Condition 1 versus Condition 4 and Condition 2 versus Condition 3 , where, in both cases, the only difference was the stimulus correlated with the second component. Note that in both comparisons, 2 subjects first received the interdimensional discrimination and the other 2 first received the intradimensional discrimination. Averaged over both comparisons, the differences in response rates for the two discriminations were 52.3 (yellow vs. vertical lines) versus 31.6 (horizontal vs. vertical lines) for $S-24,34.5$ versus 25.3 for $S-25,38.0$ versus 37.4 for $S-26$, and 26.6 versus 14.5 for S-28. In general, therefore, differences in discrimination that did occur supported the supposition that the two line tilts were more similar than were the lines versus the diffuse yellow color, although for 1 subject (S26) there appeared to be little difference. A similar result was obtained from a discrimination index (the percentage of the total responses to the stimulus correlated with the higher reinforcement rate).

Figure 1 shows the pattern of local response rates during each component averaged over the last 10 sessions of each condition. Shown on the left side of each quadrant of the figure are the patterns for when the schedule during the variable component was VI $30 \mathrm{sec}$; shown on the right side are the patterns when the variable schedule was VI $270 \mathrm{sec}$. The solid symbols represent the conditions with the interdimensional discrimination; the unfilled symbols represent the conditions with the intradimensional discrimination.

The patterns of local responses were highly variable, both across subjects and across conditions. They are presented to illustrate that essentially all of the different types of local-rate gradients that had been reported previously were here obtained within a single study. Consider- 

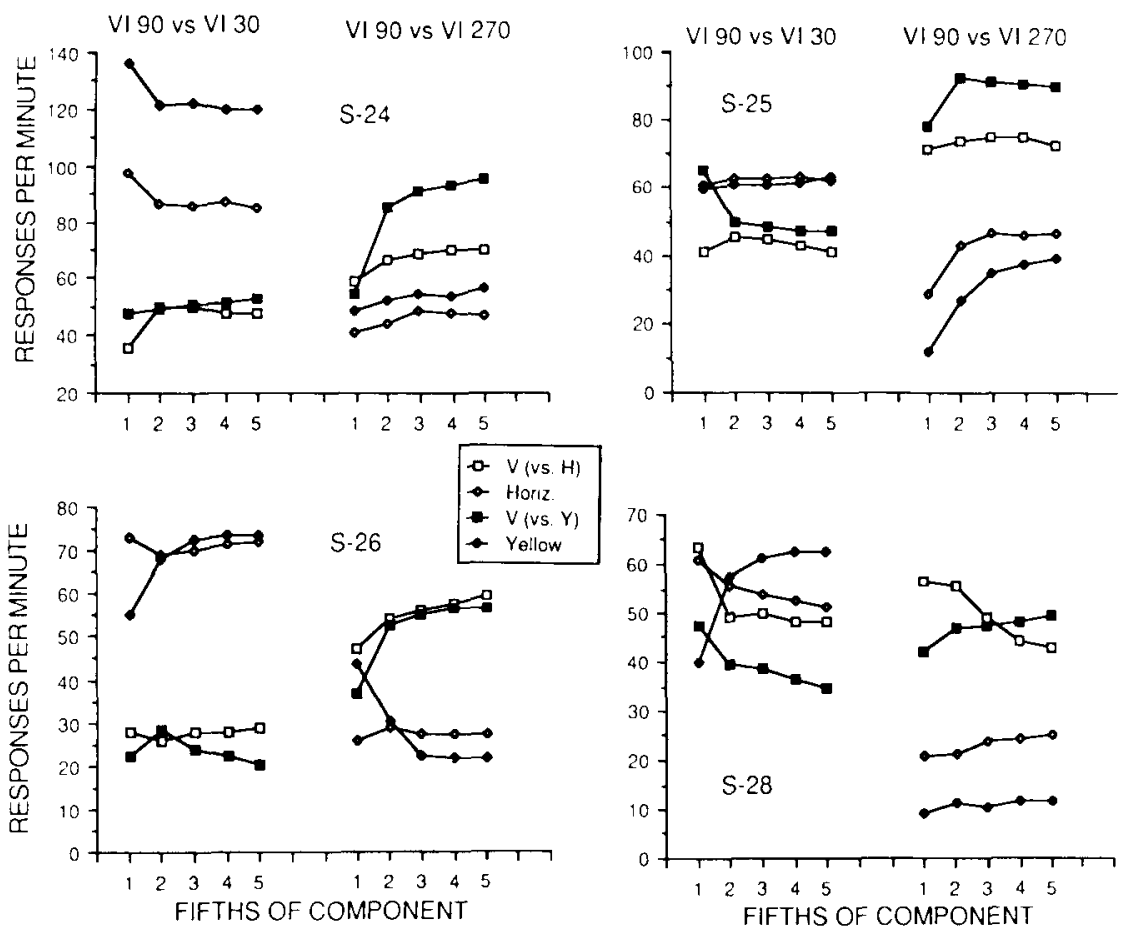

Figure 1. Local response rates in the different segments of each component averaged over the last 10 sessions of each condition. The two segments of the data for each subject correspond to the different schedules during the variable component (VI $30 \mathrm{sec}$ or VI $270 \mathrm{sec}$ ). Note that the schedule during the vertical lines was always VI 90 sec. Each segment shows the response rates in both components of the schedule, both when the discrimination was between the vertical (V) and horizontal lines (H) and when it was between the vertical lines and yellow.

ing first the components with the higher valued schedule, there were four cases with decreasing local rates consistent with positive local contrast, six cases with increasing local rates (cf. Buck et al., 1975), and six cases with either flat or nonmonotonic gradients. For the components with the lower valued schedule, there were eight cases with increasing local rates, consistent with negative local contrast, three cases with decreasing local rates, and five that were flat or nonmonotonic.

Because of the variability in the pattern of absolute local rates, the effects of similarity are difficult to assess. With respect to the higher valued component, the yellow versus vertical discrimination was more likely to produce an increasing pattern of local rate (or a notably greater slope), as occurred during the VI 90 versus VI 270 condition for all 4 subjects, and for the VI 90 versus VI 30 condition for S-26 and S-28. Differences between the two discriminations during the lower valued component were most frequently in terms of response rate rather than pattern, but differences in pattern occasionally did occur, which showed the intradimensional discrimination producing a lower response rate at component onset (VI 90 vs. VI 30 for S-24 and S-25, VI 90 vs. VI 270 for S-26).

One method for canceling at least some sources of variability is to convert the absolute response rates into relative measures. Figure 2 shows one such measure for the component in which the reinforcement schedule was held constant (vertical lines). The measure plotted is the percentage increase in response rate that occurred for the condition in which the reinforcement rate in the alternative component was lowest relative to the condition in which the reinforcement rate in the alternative component was highest, that is, the size of the contrast effect in percentage terms. In terms of the data shown in Figure 1, this measure corresponds to the difference between the functions when the schedule in the variable component was VI $30 \mathrm{sec}$ versus VI $270 \mathrm{sec}$, divided by the rate when the alternative reinforcement was VI $30 \mathrm{sec}$. Separate calculations were performed for individual segments of the component, for each separate discrimination. The results thus provide a measure of how the size of the contrast effect changed as a function of the component segment. Note that the percentage change measure is not differentiated with respect to positive and negative contrast procedures. That is, in some cases the reinforcement rate in the alternative component was increased, and in other cases it was decreased. Although it is possible that positive and negative contrasts are affected differently by stimulus similarity, there is no evidence that the two types of manipulations are functionally dissimilar for any experimental variable heretofore studied with free-operant procedures, so that distinction is ignored here.

Figure 2 shows the pattern of change not only for the last 10 sessions of training, but also for the first 5 sessions 

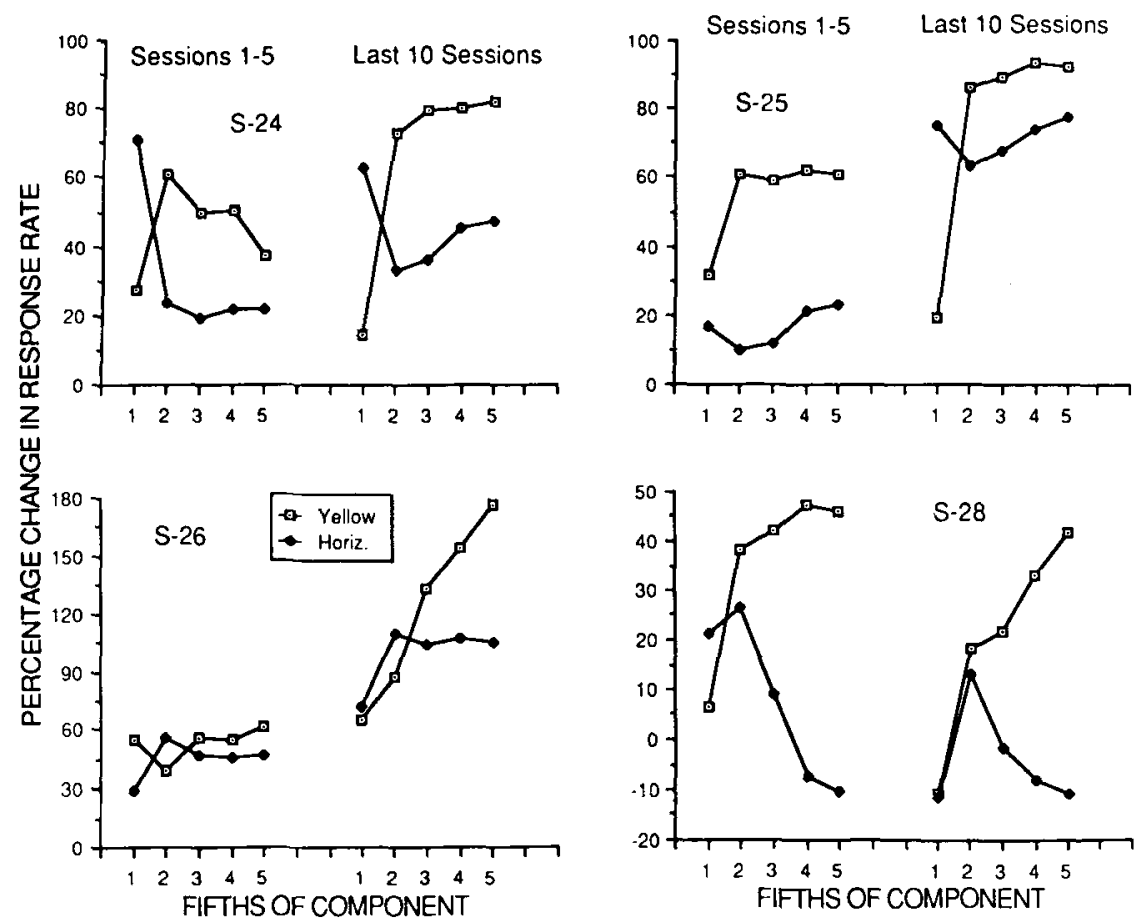

Figure 2. The percentage change in response rates in the constant component (always the vertical lines) as a result of the change in reinforcement schedules during the variable component. The measure was derived by subtracting the rate when the alternative schedule was VI 30 sec from the rate when the alternative schedule was VI $270 \mathrm{sec}$, and dividing that difference by the former value. Separate functions are for conditions when the stimulus during the variable component was either yellow or the horizontal lines. The different segments are from the first 5 sessions after the schedule change and the last $\mathbf{1 0}$ sessions of each condition.

after the change in schedules, in order better to detect any local contrast effects that might have occurred early and then dissipated. In general, the effects of continued training were to increase the overall level of contrast and to increase differentially the magnitude of the change near the end of the component. For S-24 and S-25, there were local contrast effects during the intradimensional discrimination that were evident during the first 5 sessions and continued throughout training. A similar pattern occurred for the intradimensional discrimination for S-28, although its aberrant response rate during the first component segment of the last 10 sessions makes its pattern difficult to interpret. Note that the 1 subject that did not show local contrast with the intradimensional discrimination (S-26) showed very little difference in the degree of discrimination for the two types of discrimination, suggesting that, for it, the vertical versus horizontal lines were highly dissimilar. There was no clear evidence of local contrast with the interdimensional discrimination, although S-26 did show a small local contrast effect during the first 5 sessions, which disappeared with training. Note that the order of training might have been an important variable, inasmuch as S-24 and S-25 first received the intradimensional discrimination, whereas S-26 and S-28 first received interdimensional discrimination.

The above observations were tested for reliability by submitting the data in Figure 2 to a three-way ANOVA (stimulus $\times$ stage of training $\times$ component segment) using a .05 confidence level. The effect of stimulus was significant $[F(1,3)=72.6]$, as was the effect of component segment $[F(4,12)=5.72]$. The effect of stage of training was not significant $[F(1,3)=2.61]$. The interaction between stimulus and component segment was significant $[F(4,12)=4.96]$, as was the interaction between stage of training and component segment $[F(4,12)=12.42]$. The remaining interactions were not significant.

Because of the significant interaction terms, separate two-way ANOVAs (stimulus $\times$ component segment) were conducted on the first 5 sessions and the last 10 sessions. For the analysis of the first 5 sessions, the effect of stimulus was significant $[F(1,3)=10.24]$ but the effect of component segment $(F<1)$ and their interaction $(F=1.96)$ were not. For the last 10 sessions, the effect of stimulus was significant $[F(1,3)=15.78]$, as were the effect of component segment $[F(4,12)=11.23]$ and the interaction term $[F(4,12)=6.75]$. The analysis of simple effects for the last 10 sessions showed that the effect of stimulus was significant for Segments 3,4 , and 5 , but not for Segments 1 and 2 ( $F$ values $=3.69, .78$, $36.37,37.08$, and 12.8 , all with $d f=1,3$ ). The effect of component segment was significant for the yellow versus vertical discrimination $[F(4,12)=14.23]$ but not for the discrimination between horizontal versus vertical $(F<1)$. The statistical analysis thus confirms that the 
contrast effect was greater with the easier discrimination, and that the type of local-rate gradient was systematically different as well, with the easier discrimination producing an increasing contrast effect toward the end of the component.

Previous investigations of the effects of stimulus similarity on the pattern of contrast effects (e.g., White et al., 1984) have generally not used percentage change in response rate as the dependent variable. Instead, they have analyzed their results in terms of the generalized matching law, as given by Equation 1, in which the degree of interaction is given by the exponent, $a$. (The values of $B$ refer to the response rates in the two components, the values of $R$ to the reinforcement rates, and the value of $b$ to any bias toward one or the other component independent of the reinforcement rates.)

$$
B_{1} / B_{2}=b\left(R_{1} / R_{2}\right)^{a} \text {. }
$$

To provide a comparable analysis for the present results, Equation 1 was fitted to the data from the last 10 sessions of each condition for individual subjects. Because there were only two data points for each discrimination for each subject, an application of Equation 1 in the present study entails no index of the reliability of the fits, because the error term is eliminated when a two-parameter equation is fit to only two data points. Although this would be a serious problem if the goal of the analysis were to test the applicability of Equation 1 to the present data, the present analysis assumes that applicability because of previous work, and uses Equation 1 only as a method of summarizing the data. Note also that the fits to the individual component segments used the reinforcement rates from the entire component, since reinforcement rates for individual segments were not recorded.

The results of the analysis, in terms of the value of $a$, are shown in Figure 3. As with the percentage change measure, the largest degree of interaction occurred for the easier discrimination and there were systematic differences in the pattern of interaction across the component segments. For the discrimination of yellow versus vertical, 3 of the 4 subjects had an increasing value of $a$ across the component; the 4th (S-25) produced the opposite gradient. For the discrimination of horizontal versus vertical, 3 of the 4 subjects had a decreasing value of $a$ across the component; the 4th (S-26) had a flat gradient. The more frequent occurrence of decreasing gradients is notably different from the patterns seen in Figure 2 . The apparent reason for this is that the value of $a$ reflects the pattern of rate changes in both components of the multiple schedule. Local-rate patterns characterized by local contrast effects were more frequent in the component with the variable schedule, as can be ascertained by a detailed inspection of Figure 1.

The results shown in Figure 3 were analyzed statistically with a two-way ANOVA (stimulus $x$ component segment). The stimulus variable only approached significance $[F(1,3)=7.87, p<.07]$; the component segment was not significant $(F<1)$, but the interaction term was $[F(4,12)=3.27]$. The statistical analysis thus confirms the analysis that the pattern of interaction was sig-
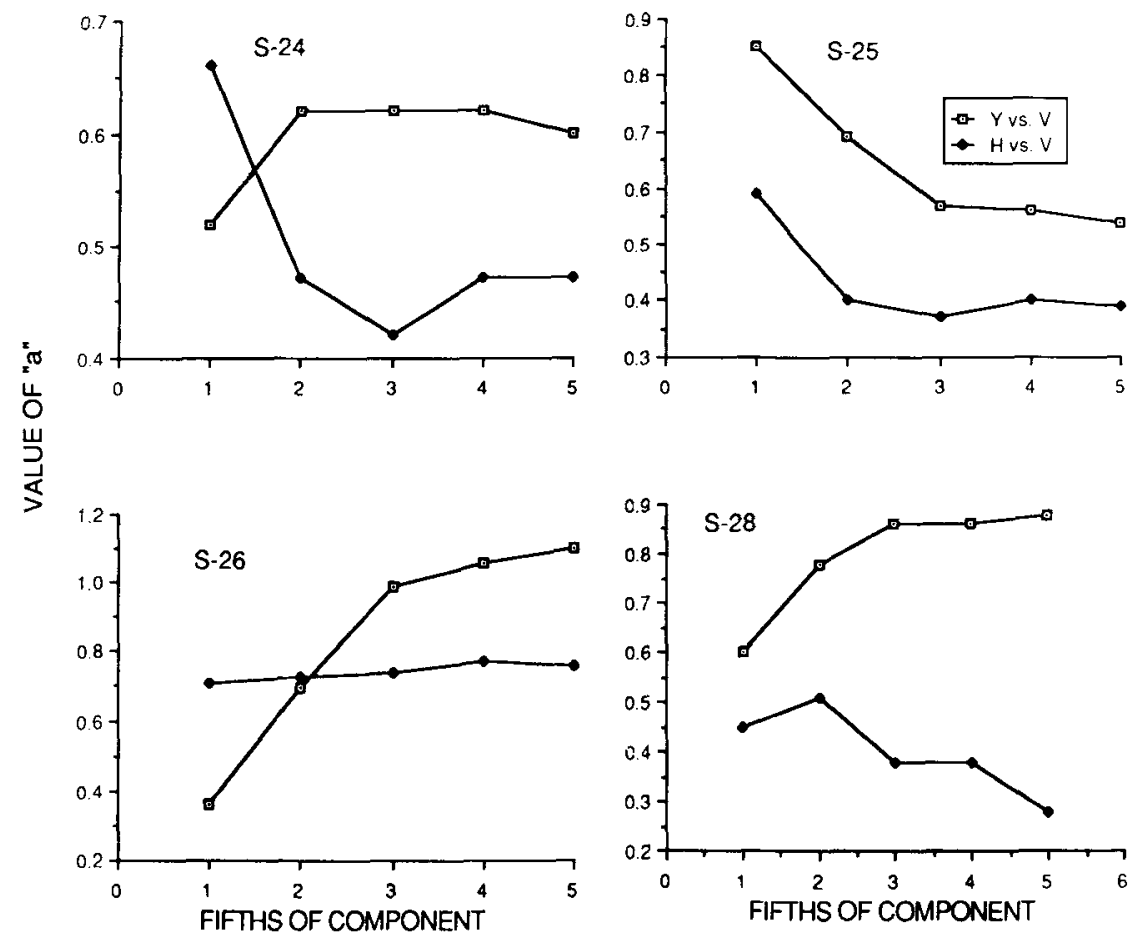

Figure 3. Value of $a$ from fits of Equation 1 to individual subjects. Data are from the last 10 sessions of each condition. 
nificantly different for the two levels of stimulus similarity, even when the generalized matching law was used as the measure of change.

\section{Discussion}

The major finding of the present study was that the effects of stimulus similarity on interactions in a multiple schedule depend upon the type of contrast effect at issue. If the degree of molar contrast is considered, as shown in Figure 2, greater contrast occurs, the more dissimilar the discriminative stimuli correlated with the two components of the schedule, a finding similar to that of several previous studies (Mackintosh et al., 1972; Malone, 1976; White et al., 1983, 1984). Moreover, the larger average size of the contrast effect is manifested primarily in terms of increases in response rate near the end of the component. Such effects are consistent with previous work showing that steady-state contrast is primarily "anticipatory" in nature, being a function of variations in the following schedule of reinforcement (Williams, 1979, 1981). However, if only local contrast at the onset of a component is considered, contrast is not larger with more dissimilar stimuli, but is, as Figure 2 reveals, larger (but not statistically reliable) at component onset with the more similar stimuli. Greater local contrast with more similar stimuli is also consistent with the findings of previous studies (Blough, 1980, 1983).

An unexpected feature of the present analysis is that the nature of the gradient reflecting the degree of interaction depended upon the type of analysis. When the contrast effects in the various component segments were calculated in terms of the percentage increase in response rate (Figure 2), the gradients were most frequently increasing in nature. But when the results were analyzed in terms of the generalized matching law (Figure 3), the largest degree of schedule interaction occurred most frequently at component onset, although an increasing interaction across component segments was still the most typical finding with the interdimensional discrimination. The apparent reason for this difference is that the description in terms of the generalized matching law uses information from both the unchanged component and the component with the variable schedule. Schedule interactions in the unchanged component were most frequently at their maximum just preceding component transition, whereas those during the variable component were often greatest just after component onset. As reported previously by McLean and White (1981), the component with the variable schedule appears to be more sensitive to local contrast effects.

The disparity between the outcomes of the different types of analyses is relevant to a conflict between the present study and the findings of previous studies, which have shown that the greater degree of molar contrast with greater stimulus dissimilarity is manifested primarily by increased schedule interactions just after component transition (White et al., 1983, 1984). Such studies used, as their measure of schedule interaction, the value of $a$ from fits by Equation 1, with the result that high values of $a$ near the component onsets were a primary portion of the overall schedule interactions. A similar pattern was found here for the majority of subjects during the intradimensional discrimination, and for 1 subject with the interdimensional discrimination. Given that the previous studies have always used intradimensional discriminations, the present findings are largely in agreement with their results. The only conflict with the previous findings is that the values of $a$ generally increased across the component segments with the easier discrimination, which presumably is due to the use of a different portion of the range of stimulus similarity. That is, with increasingly dissimilar stimuli, the contribution of local contrast to molar contrast is diminished, so that the contribution of anticipatory contrast becomes more apparent.

One caveat regarding the present findings is that they do not allow a conclusion about whether the effects of stimulus similarity apply to positive contrast, to negative contrast, or both. The use of only two pairs of reinforcement schedules entails that the distinction between positive and negative contrast is lost, because whether the difference between the pairs of conditions is viewed as positive or negative contrast is entirely a function of the order of conditions. Although no previous data demonstrate that the functional properties of positive and negative contrast differ in any important way, some previous data (Blough, 1983) have suggested that the two types of contrast may vary in their sensitivity to the stimulussimilarity variable, although the direction of the effect was the same in both cases.

\section{EXPERIMENT 2}

The results of Experiment 1 suggest that there are at least two components of schedule interactions that may be affected differently by stimulus similarity. Anticipatory contrast was clearly increased by greater stimulus dissimilarity, inasmuch as contrast increased as time into the component increased. The effect on local contrast was less clear, with some subjects showing an enhancement of local contrast with greater similarity and others not (see the different subjects in Figure 2). Unfortunately, any conclusions about the effects of stimulus similarity on different types of contrast are limited by the interpretation of gradients of local response rate. That is, anticipatory contrast was indexed by increasing gradients, whereas local contrast was indexed by decreasing gradients, with the implicit assumption that the effects could be detected independently. In fact, the opposition between the two types of local-rate patterns means that the effects may be in competition, and it is possible that one or the other effect may dominate, thus obscuring any evidence of the remaining effect. Moreover, local-rate gradients are often highly variable and determined by features of the procedure other than the changes in reinforcement rates (e.g., carryover effects from the preceding condition).

What appeared to be needed was an assessment of local response-rate patterns in which the influences of the different sources of contrast had been separated. Previ- 
ous work had dissociated the effects of variations in reinforcement from the preceding component, as opposed to those from the following component, by using multicomponent schedules in which different orders of presentation allowed for a functional separation of the two types of effects (Williams, 1979, 1981). In Experiment 2, a similar tactic was adopted while stimulus similarity was varied. The same subjects from Experiment 1 were continued, but now with a recycling, three-component schedule in which reinforcement rate was varied only during the middle component. Thus, changes in response rate during the first component would be due to anticipatory contrast, whereas those in the third component would be due to variations in the preceding component, which presumably had local contrast as a major component. In all cases, the stimulus correlated with the middle component was the horizontal lines. In some sequences, the vertical lines occurred in the first component and the yellow keylight in the third; in other sequences, the order was reversed. The rationale was to isolate functionally the effects of similarity on the different types of contrast.

\section{Method}

The subjects and apparatus were the same as in Experiment 1. For all conditions, a recycling three-component multiple schedule was used in which component duration was $30 \mathrm{sec}$. The schedule during Components 1 and 3 was always the same VI 90-sec distribution used in Experiment 1. The schedule during Component 2 was either VI $30 \mathrm{sec}$ or VI $270 \mathrm{sec}$, also the same schedules as used in Experiment 1. Conditions 1 and 4 had a VI 30-sec schedule during Component 2, and Conditions 2 and 3 had a VI 270-sec schedule. Training during each session continued until each component had 40 presentations.

For S-24 and S-25, the initial sequence of stimulus presentation was vertical lines followed by horizontal lines followed by yellow; this was then changed during Conditions 3 and 4 to yellow followed by horizontal lines followed by vertical lines. S-26 and S-28 received the opposite order of presentation. Each condition was presented for a total of 30 sessions.

\section{Results}

Before proceeding to an analysis of the schedule interactions, it is important again to validate that the stimuli differed in discriminability. Accordingly, the difference between response rates to the horizontal lines and those to the other two stimuli was calculated for the last 10 sessions of each condition. The differences (averaged over all four conditions) for the yellow versus horizontal and for vertical versus horizontal discriminations were, respectively, 71.3 versus 63.7 for $S-24,18.0$ versus 8.3 for $S-25,52.1$ versus 45.1 for $S-26$, and 4.5 versus 4.2 for S-28. Thus, all subjects had a larger difference for the interdimensional discrimination, although 1 subject (S28) had relatively little discrimination between either pair of stimuli.

Figure 4 shows the local response rates during the components before and after the middle component in which reinforcement rate was varied. Shown for each location are the four local-rate gradients produced by each combination of stimulus (yellow vs. vertical lines) and rein- forcement rate during the middle component (VI $270 \mathrm{sec}$ vs. VI $30 \mathrm{sec}$ ). Considering, first, the response rates in the before component when the schedule during the horizontal lines was VI $270 \mathrm{sec}$, these gradients were increasing for all 4 subjects when the before stimulus was yellow (although only slightly so for S-28) and slightly increasing for 3 of the subjects when the stimulus was the vertical lines (S-25 was the exception). When the schedule during the middle component was VI $30 \mathrm{sec}$, decreasing gradients occurred for all 4 subjects with the yellow stimulus and for 2 subjects (S-24 and S-28) with the vertical lines. Thus, the pattern in the before component was characterized by anticipatory contrast as response rate increased prior to a lower rate of reinforcement and decreased prior to a higher rate of reinforcement. Considering the response rates in the after component when the schedule was VI $270 \mathrm{sec}$, decreasing gradients (characteristic of positive local contrast) occurred for 2 subjects (S-24, S-25) with the yellow stimulus and also for 2 subjects (S-24, S-28) with the vertical lines. When the schedule was VI $30 \mathrm{sec}$, increasing gradients (characteristic of negative local contrast) occurred for 3 subjects with the yellow stimulus (the exception was S-24) and for the same 3 subjects with the vertical lines.

The effects of stimulus similarity are seen more clearly in Figure 5, which shows the size of the contrast effects for individual subjects, again calculated in terms of the percentage increase in response rate by taking the difference in the rates during the constant components corresponding to the two different reinforcement rates in the variable component and dividing that difference by the rate when the variable schedule was VI $30 \mathrm{sec}$. Figure 5 shows the results of this analysis, presented separately for when the stimuli occurred first in the sequence (before the horizontal lines) and last in the sequence (after the horizontal lines). As can be seen, contrast effects were generally larger during the before components, although this effect was true for all subjects only with the yellow stimulus. Most importantly, the pattern of response rate was quite different within the before versus after components, as the local rates generally increased in the former and decreased in the latter. The effects of stimulus similarity in the two locations also differed: all subjects had a larger molar contrast effect with the more dissimilar stimulus during the before component, whereas the effects during the after component were mixed, with 2 subjects showing greater contrast effects with the more similar stimulus (S-26 and S-28) and 2 showing greater contrast effects with the less similar stimulus (S-24, S-25).

The data shown in Figure 5 were analyzed separately for the before and after components. Both were submitted to two-way ANOVAs (stimulus $\times$ component segment) using a .05 level of significance. For the before analysis, the effect of stimulus was significant $[F(1,3)=$ 10.54], as were the effect of component segment $[F(4,12)$ $=6.87]$ and their interaction $[F(4,12)=4.06]$. Thus, not only was there greater contrast with the more dissimilar stimulus, but that effect was larger the nearer the end 

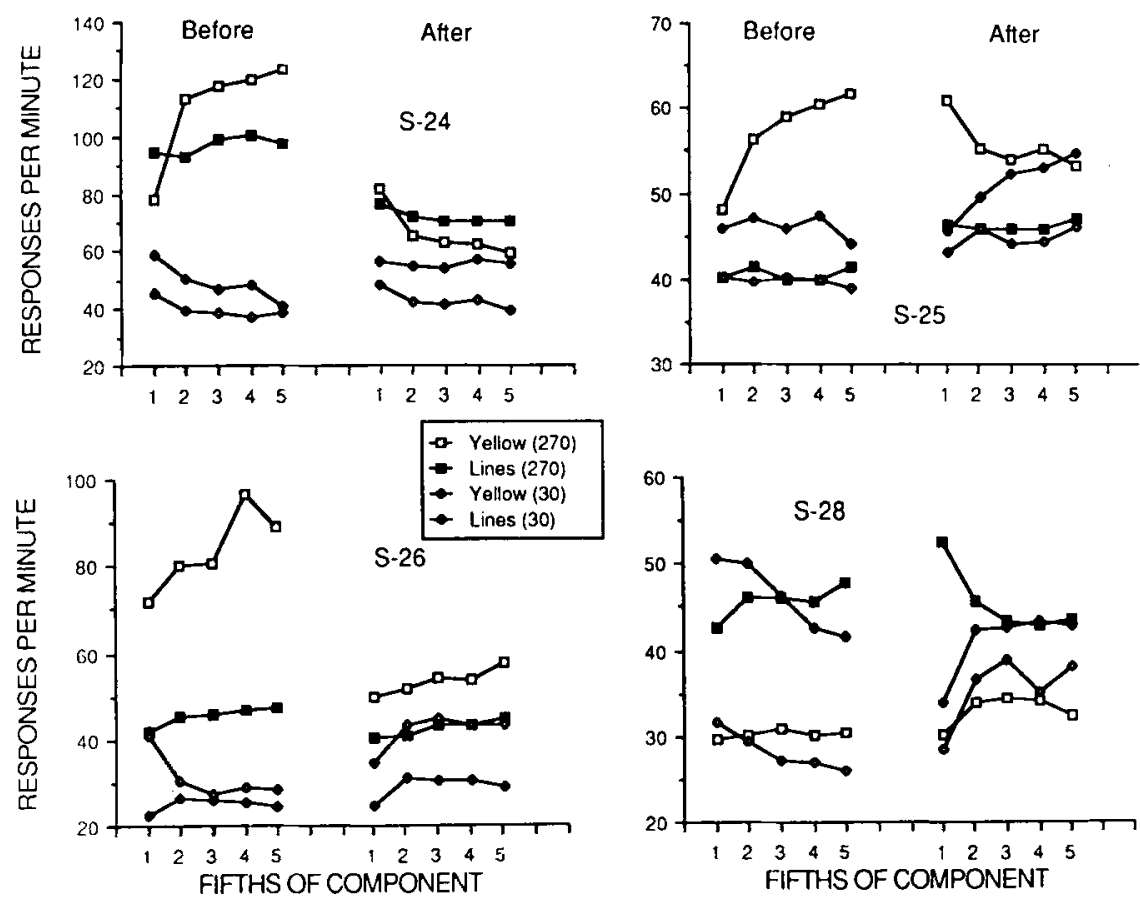

Figure 4. Local response rates from each constant component for individual subjects. All data are from the last 10 sessions of training on each condition. The left portion of the data for each subject corresponds to the component preceding the middle component with the variable schedule. The right portions correspond to the component after the middle component. The four functions of each portion correspond to the different combinations of the different stimuli correlated with the components and of the value of the VI schedule in the middle component (270 or $30 \mathrm{sec}$ ). Note that different subjects received different orders of these two schedule values, as noted in the Method section of Experiment 2.
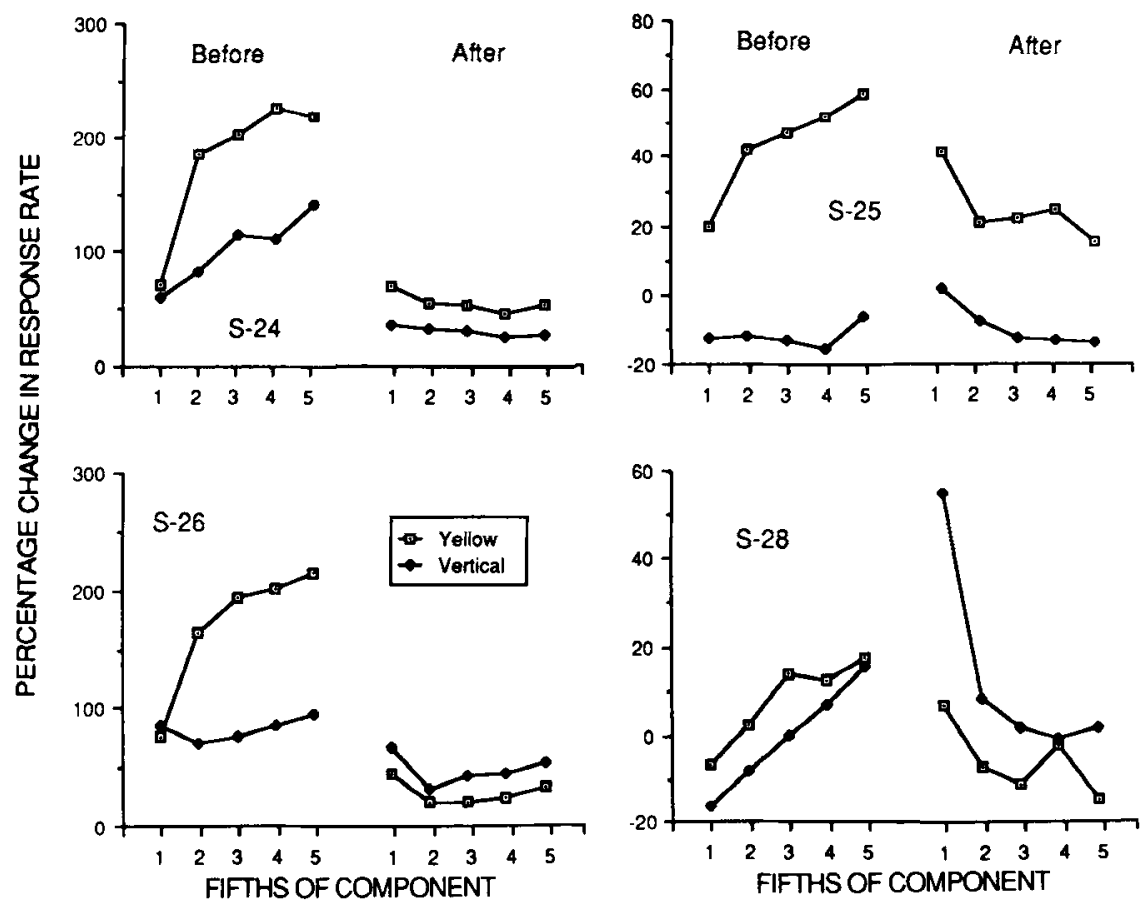

Figure 5. The size of the contrast effect, expressed in terms of the percentage change in response rate, during the vertical lines and during yellow, subdivided into component segments and calculated separately as a function of the location of the stimulus relative to the horizontal lines. Contrast was assessed by the difference in response rates between the last 10 sessions of successive conditions. 
of the before component (and the closer to the beginning of the constant component). Analysis of the simple effects further revealed that the component segment variable was significant even when the vertical lines was the preceding stimulus $[F(4,12)=3.29]$.

Analysis of the contrast effects in the after component revealed that the stimulus variable was not significant $(F<1)$, that the effect of component segment was significant $[F(4,12)=9.90]$, and that the interaction was not significant $(F<1)$. Analysis of the simple effects of component segment revealed that it was significant for both yellow $(F=10.96)$ and the vertical lines $(F=4.52$; both tests with $d f=4,12$ ). Thus, there was no reliable effect of stimulus on either the size or pattern of local contrast, and for both levels of stimulus similarity the contrast effect was largest just after component transition and then decreased.

\section{Discussion}

The results of Experiment 2 were generally consistent with those of Experiment 1 in showing how stimulus similarity affects different forms of behavioral contrast. For anticipatory contrast, indexed in the present case by rate changes in the component preceding the horizontal lines in which reinforcement rate was varied, the size of the contrast effect was greater with more dissimilar stimuli. The size of the contrast effect also increased with time into the component (as the variable schedule came closer to onset), and the degree of this increase was also facilitated by more similar stimuli. For local contrast, indexed by rate changes in the component following the horizontal lines, the effects of stimulus similarity were more variable across subjects. Regardless of stimulus similarity, the largest contrast effects occurred just after component transition, although for some subjects this effect was larger when the stimulus transition involved more similar stimuli, whereas for others it was larger when it involved less similar stimuli.

The procedure used in Experiment 2 has the major advantage of functionally separating local contrast from anticipatory contrast as determinants of local response-rate gradients. Had only two components been used, as in Experiment 1 and all previous examinations of stimulus similarity, the separate effects seen in Figure 5 would necessarily combine to produce an overall local-rate function highly variable for different subjects because of the different relative magnitudes of the two types of contrast. Thus, the subjects with large anticipatory contrast effects relative to the size of local contrast would be expected to produce increasing local-rate gradients, whereas those with the reverse pattern would be expected to produce decreasing local-rate gradients. It should therefore not be surprising that increasing stimulus dissimilarity was more likely to produce an increasing type of local rate gradient, because greater dissimilarity increased anticipatory con- trast more than local contrast for all subjects (and, indeed, had the opposite effect on local contrast for some subjects). The implication is that there is no one effect of stimulus similarity on molar contrast effects, since the overall effect will be a function of the relative contribution of the different types of contrast to the total.

The different effects of stimulus similarity on anticipatory versus local contrast are important because they strongly suggest that the two types of effects depend upon different mechanisms. As discussed in the introduction, local contrast has been most often interpreted in terms of Pavlovian processes involved in stimulus discrimination, which are often transitory unless similar stimuli are involved. Anticipatory contrast, on the other hand, appears to be enhanced by continued training and more dissimilar stimuli, which would be expected if it is controlled by the relative rate of reinforcement. That is, the more clearly the subject can discriminate the prevailing reinforcement conditions in the alternative components of the schedule, the more evident anticipatory contrast will be.

To the extent that the two forms of contrast do depend upon different mechanisms, contrast studies are likely to produce variable outcomes, depending upon the exact particulars of the procedures. For example, it is unknown to what extent the two types of contrast contribute differently for different species (e.g., rats vs. pigeons) or for the effects of important parameters such as component duration. The present results thus suggest that it is imperative that studies of contrast use procedures that allow for dissociation between the different types of contrast, such as the three-component schedule used here or even more complex multiple schedules such as those used previously by Farley (1980) and Williams (1979).

The most important theoretical implication of the present results is that they challenge any theory of contrast that relies entirely on relative rate of reinforcement as the controlling variable. For example, Williams and Wixted (1986) presented a quantitative model of contrast based on the assumption that the response-strengthening effects of reinforcement are inversely related to the context of reinforcement in which it occurs, and that the context is calculated as the weighted average of the various sources of reinforcement in the situation. Although that analysis can accurately describe the results of existing contrast studies if the weights given to the reinforcement conditions following the target component are different from those given to the reinforcement conditions preceding it, the implicit assumption that the two effects are based on a similar comparison process seems to be fundamentally misguided. That is, effects of reinforcement in the preceding component appear to be functionally dissimilar from those in the following component, and thus demand a separate analysis. Rather than conceptualizing contrast as the result of a comparison process between molar rates of reinforcement during the stimulus versus its context, 
the present results suggest that a more profitable direction will be to investigate the different mechanisms underlying the two different types of contrast that have been isolated.

\section{REFERENCES}

BLough, P. M. (1980). Behavioral and dimensional contrast in rats. Journal of the Experimental Analysis of Behavior, 33, 345-357.

BLough, P. M. (1983). Local contrast in multiple schedules: The effect of stimulus discriminability. Journal of the Experimental Analysis of Behavior, 39, 427-435.

Boneau, C. A., \& AXelrod, S. (1962). Work decrement and reminiscence in pigeon operant conditioning. Journal of Experimental Psychology, 64, 353-354.

Buck, S. L., Rothstein, B., \&illiams, B. A. (1975). A reexamination of local contrast in multiple schedules. Journal of the Experimental Analysis of Behavior, 24, 291-301.

FARLEY, J. (1980). Automaintenance, contrast, and contingencies: Effects of local vs. overall and prior vs. impending reinforcement context. Learning \& Motivation, 11, 19-48.

Fleshler, M., \& Hofman, H. S. (1962). A progression for generating variable-interval schedules. Joumal of the Experimental Analysis of Behavior, 5, 529-530.

GREEN, L., \& RACHUIN, H. (1975). Economic and biological influences on a pigeon's key peck. Journal of the Experimental Analysis of Behavior, 23, 55-62.

Hamilton, B. E., \& Silberberg, A. (1978). Contrast and autoshaping in multiple schedules varying reinforcer rate and duration. Journal of the Experimental Analysis of Behavior, 30, 107-122.

HeArst, E. (1969). Excitation, inhibition, and discrimination learning. In N. J. Mackintosh \& W. Honig (Eds.), Fundamental issues in as sociative learning (pp. 1-41). Halifax: Dalhousie University Press.

MaCKINTOSH, N. J., LitTLE, L., \& LoRD, J. (1972). Some determinants of behavioral contrast in pigeons and rats. Learning \& Motivation, $3,148-161$

MALONE, J. C. (1976). Local contrast and Pavlovian induction. Journal of the Experimental Analysis of Behavior, 26, 425-440.

McLeAN, A. P., \& WiTE, K. G. (1981). Undermatching and contrast within components of a multiple schedule. Journal of the Experimental Analysis of Behavior, 35, 283-291.

Nevin, J. A., \& Shettleworth, S. J. (1966). An analysis of contrast effects in multiple schedules. Journal of the Experimental Analysis of Behavior, 9, 305-315.

Rachlin, H. C. (1973). Contrast and matching. Psychological Review, 80, 217-234.

SCHWARTZ, B. (1978). Stimulus-reinforcer contingencies and local behavior contrast. Journal of the Experimental Analysis of Behavior, 29, 297-308.

Schwartz, B., Gamzu, E. (1977). Pavlovian control of operant behavior: An analysis of autoshaping and its implications for operan conditioning. In W. K. Honig \& J. E. R. Staddon (Eds.), Handbook of operant behavior (pp. 53-97). New York: Prentice-Hall.

SPEALMAN, R. D. (1976). Interactions in multiple schedules: The role of the stimulus-reinforcer contingency. Journal of the Experimental Analysis of Behavior, 26, 79-93.

Terrace, H. S. (1966). Stimulus control. In W. K. Honig (Ed.), Operant behavior: Areas of research and application (pp. 271-344). New York: Appleton-Century-Crofts.
White, K. G., PiPe, M.-E., \& Mclean, A. P. (1983). Dimensional stimulus control of multiple schedule performance. Behavior Analysis Letters, 3, 51-57.

White, K. G., PiPe, M.-E., \& McLean, A. P. (1984). Stimulus and reinforcer relativity in multiple schedules: Local and dimensional effects on sensitivity to reinforcement. Journal of the Experimental Analysis of Behavior, 41, 69-81.

White, K. G., PIPE, M.-E., \& Redman, S. (1985). Temporal proximity and reinforcement sensitivity in multiple schedules. Journal of the Experimental Analysis of Behavior, 44, 207-215.

Williams, B. A. (1974). The role of local interactions in behavioral contrast. Bulletin of the Psychonomic Society, 4, 543-545.

Williams, B. A. (1976a). Behavioral contrast as a function of the temporal location of reinforcement. Journal of the Experimental Analysis of Behavior, 26, 57-64.

WiLliams, B. A. (1976b). Elicited responding to signals for reinforcement: The effects of overall versus local changes in reinforcement probability. Journal of the Experimental Analysis of Behavior, 26, 213-220.

Williams, B. A. (1979). Contrast, component duration, and the following schedule of reinforcement. Journal of Experimental Psychology: Animal Behavior Processes, 5, 379-396.

Williams, B. A. (1981). The following schedule of reinforcement as a fundamental determinant of steady-state contrast in multiple schedules. Joumal of the Experimental Analysis of Behavior, 35, 293-310.

Williams, B. A. (1982). Do interactions in multiple and concurrent schedules have a common basis? In M. L. Commons, R. J. Herrnstein, \& H. Rachlin (Eds.), Quantitative analyses of behavior: Vol. 2. Matching and maximizing accounts (pp. 281-302). Cambridge, MA Ballinger Press.

Williams, B. A. (1983). Another look at contrast in multiple schedules. Journal of the Experimental Analysis of Behavior, 39, 345-384. Williams, B. A., \& WIXTED, J. T. (1986). An equation for behavioral contrast. Joumal of the Experimental Analysis of Behavior, 45, 47-62.

\section{NOTE}

1. The present terminology was chosen to be theoretically neutral. In its most general sense, behavioral contrast is defined by an inverse relationship between response rate in a target component and the reinforcement rate in some alternative component successively available. Such a definition encompasses both positive and negative contrast effects, which are defined by different procedures. With positive contrast, increases in response rate occur when the alternative reinforcement is decreased (usually relative to a baseline in which reinforcement rate in the two components are equal); with negative contrast, decreases in response rate occur when the alternative reinforcement is increased (again relative to the baseline with equal reinforcement rates). Positive contrast and negative contrast have both been studied separately in previous studies of both local and molar contrast. Whether the two types of contrast correspond to different behavioral processes is still an open question, but the present study ignores the distinction by assuming that the functional relationship between response rate and alternative reinforcement rate is continuous and thus independent of the direction in which the alternative reinforcement rate is changed.

(Manuscript received August 4, 1987; revision accepted for publication November 23, 1987.) 